\title{
Combination therapy with $\alpha$-galactosylceramide and a Toll-like receptor agonist exerts an augmented suppressive effect on lung tumor metastasis in a mouse model
}

\author{
TATSUYA ANDO, HIROYASU ITO, YUKO ARIOKA, HIDEYUKI OGISO and MITSURU SEISHIMA \\ Department of Informative Clinical Medicine, Gifu University Graduate School of Medicine, Gifu 501-1194, Japan
}

Received October 2, 2014; Accepted November 4, 2014

DOI: $10.3892 /$ or.2014.3634

\begin{abstract}
GalCer), which is a natural killer T (NKT) cell ligand, has been reported to exert therapeutic effects against cancer in humans and mice. Toll-like receptor (TLR) agonists systemically or locally boost antitumor efficacy in mouse cancer models. In our previous study, the co-administration of GalCer and a TLR agonist synergistically enhanced interferon- $\gamma($ IFN- $\gamma$ ) production in mouse splenocytes in vitro and in vivo. The increased IFN- $\gamma$ production promoted a tumor antigen-specific Th1 response. Therefore, co-treatment with GalCer and a TLR agonist is expected to exert an enhanced antitumor effect. In the present study, we examined the effect of GalCer and lipopolysaccharide (LPS) combination therapy in a mouse lung-metastasis model. GalCer and LPS combination therapy markedly decreased the number of lung metastatic tumor nodes. Co-treatment with GalCer and LPS enhanced the mRNA expression of CXCL9 and CXCL10 in mediastinal lymph nodes (MLNs) and increased the number of $\mathrm{CD}^{+}$cells in the MLNs. Furthermore, the depletion of $\mathrm{CD}^{+} \mathrm{T}$ cells canceled the antitumor effect of GalCer and LPS combination therapy. Thus, GalCer and LPS combination therapy significantly enhanced tumor antigen-specific immune responses and suppressed tumor growth in a mouse lung-metastasis model.
\end{abstract}

\section{Introduction}

Lung metastasis is the leading cause of cancer-related death worldwide. Although treatment methods such as surgery, irradiation and chemotherapy have improved, the prognosis of patients with lung metastasis remains unsatisfactory, and new therapeutic strategies are needed. Immunotherapy may represent a new therapeutic strategy for lung metastasis $(1,2)$. The goal of immunotherapy for lung metastasis is to enhance the

Correspondence to: Dr Hiroyasu Ito, Department of Informative Clinical Medicine, Gifu University Graduate School of Medicine, 1-1 Yanagido, Gifu 501-1194, Japan

E-mail: hito@gifu-u.ac.jp

Key words: $\alpha$-galactosylceramide, lipopolysaccharide, NKT, IFN- $\gamma$, cytotoxic T lymphocytes, lung metastasis weakened host immune response against tumors using specific and/or non-specific immune stimulants.

$\alpha$-galactosylceramide (GalCer) is a glycolipid that binds to CD1d, and it is recognized by invariant natural killer $\mathrm{T}$ (iNKT) cells. Injection with GalCer enhances the activation of NKT cells, resulting in the rapid release of both Th1 and Th2 cytokines, thereby eliciting a downstream cascade of activation that spreads to dendritic, NK and B cells (3-5). These activation cascades powerfully induce adaptive immunity. Recent studies have revealed the mechanism of GalCer-induced iNKT cell activation in immune responses to tumors and microbes, as well as in the suppression of autoimmune diseases (6-9).

Toll-like receptors (TLRs) recognize specific molecular signatures called pathogen-associated molecular patterns that are present in pathogens. Members of the TLR ligand class of adjuvants, including Pam3Cys (TLR-2), poly-IC (TLR3), lipopolysaccharide (LPS) (TLR4), imiquimod (TLR7) and CpG-ODN (TLR9), induce antigen-presenting cell maturation and the production of inflammatory cytokines, favoring effector $\mathrm{T}$ cell responses and restricting Treg expansion (10), indicating that TLR signaling induces the activation of immune responses. TLR3 agonists with variable efficiency have been used previously as adjuvants in the treatment of cancer, with the aim of inducing an IFN-mediated antitumor immune response (11). TLR4 stimulation can overcome CD8 ${ }^{+}$ $\mathrm{T}$ cell tolerance and eradicate established tumors (12). TLR7 and TLR9 ligands induce cancer cell death by enhancing cytotoxic $\mathrm{NK}$ and $\mathrm{CD}^{+} \mathrm{T}$ cell activation of anti-angiogenic factors and by promoting the production of anti-angiogenic factors (13). The antitumor effects of TLR activation in diverse cancer subtypes are postulated to proceed via several parallel mechanisms, including potentiating innate immune responses via activation of NK cells, monocytes and macrophages; inducing the generation of tumoricidal cytokines; inducing Th1 deviation of $\mathrm{CD}^{+}{ }^{+} \mathrm{T}$ cells; augmenting CTLs; and directly inducing apoptosis in TLR-expressing tumor cells (14).

Thus, GalCer and TLR ligands have been used to treat various types of cancers in the basic and clinical settings. However, these therapies have had limited success against human cancers in many studies. We recently reported that GalCer and a TLR agonist synergistically enhanced the production of interferon- $\gamma($ IFN- $\gamma)$ and induced a robust immunological response in mice (15-18). Facilitation of IFN- $\gamma$ production indicates the enhancement of Th1 immune 
responses in hosts $(6,19,20)$. Therefore, combination therapy with GalCer and a TLR agonist induces a robust Th1 immune response in tumor-bearing hosts and suppresses tumor growth. In the present study, we demonstrated that combination therapy with GalCer and a TLR ligand suppressed tumor growth in a mouse lung-metastasis model.

\section{Materials and methods}

Mice. Male BALB/c mice at the age of $\sim 8$ weeks were obtained from Japan SLC Inc. (Hamamatsu, Japan). All animal procedures were conducted in accordance with the National Institutes of Health Guide for the Care and Use of Laboratory Animals and the Guidelines for the Care and Use of Animals established by the Animal Ethics Committee of Gifu University.

Cell lines and reagents. CT26 cells (mouse colon carcinoma) used in the present study were generously provided by Dr Hidekazu Shirota (Laboratory of Experimental Immunology, Cancer and Inflammation Program, the National Cancer Institute, Frederick, MD, USA). Synthesized GalCer was obtained from Funakoshi, Co., Ltd. (Tokyo, Japan). LPS from Escherichia coli O111:B4 was purchased from Sigma-Aldrich (St. Louis, MO, USA). CFSE was purchased from BioLegend (San Diego, CA, USA).

Tumor challenge and therapy. Mice were intravenously injected with $7.5 \times 10^{4}$ CT26 colon carcinoma cells and after 5 days, GalCer ( $1 \mu \mathrm{g} /$ mouse) was administered into the tumorbearing mice. On the next day, LPS (1 $\mu \mathrm{g} /$ mouse) was injected intravenously. Five days following LPS injection, mice were sacrificed, and their lungs were removed to count superficial metastatic nodules.

Enzyme-linked immunospot (ELISPOT) assay. Tumor-bearing mice were treated with LPS and GalCer, either alone or in combination. Single-cell suspensions were prepared from the mediastinal lymph nodes (MLNs) on day 1 after the inoculation. A total of $2.0 \times 10^{5}$ lymphocytes/well were stimulated for $14-16 \mathrm{~h}$ with $0,0.1$ or $1 \mu \mathrm{g} / \mathrm{ml}$ of $\mathrm{AH}-1$ peptide (Medical and Biological Laboratories Co., Ltd., Nagoya, Japan) in 96-well MultiScreen filter plates (Millipore, Billerica, MA, USA) pre-coated with a monoclonal rat anti-IFN- $\gamma$ antibody (R4-6A2; BD Biosciences, Franklin Lakes, NJ, USA). The plates were washed and then incubated with a biotinylated polyclonal goat anti-IFN- $\gamma$ antibody (R\&D Systems, Minneapolis, MN, USA) followed by incubation with streptavidin-alkaline phosphatase. Spots were visualized by the addition of a 5-bromo-4-chloro-3-indolyl phosphate solution (Sigma-Aldrich) and counted manually under a microscope (magnification, $\mathrm{x} 40$ ). The number of cytokine-secreting cells was determined by a single observer in a blinded manner, and all data were generated by analyzing 3 separate wells/sample.

Flow cytometry. Lymphocytes were isolated from the MLNs and lungs of tumor-bearing mice 1 day after LPS injection with or without GalCer treatment. Flow cytometry was used to evaluate the expression levels of CD4, CD8, Foxp3, CD69 and CXCR3. The isolated cells were stained with allophyco- cyanin (APC)-conjugated anti-mouse CD4 (clone RM 4-5), fluorescein isothiocyanate (FITC)-conjugated anti-mouse CD8 (clone 53-6.7), phycoerythrin (PE)-Cy7-conjugated antimouse CD69 (clone H1.2F3), PE-conjugated anti-mouse Foxp3 (clone FJK-16s) and APC-conjugated anti-mouse CXCR3 (clone CXCR3-173) (eBiosocience). The stained cells were analyzed using a FACSCanto II instrument (BD Biosciences, San Jose, CA, USA).

Quantitative reverse transcription-polymerase chain reaction $(R T-q P C R)$ analysis. RT-qPCR was used to quantify the mRNA expression levels of various genes in the MLNs. Total RNA was isolated using Isogen II reagent (Nippon Gene, Tokyo, Japan), and it was transcribed into cDNA using a High-Capacity cDNA transcription kit (Applied Biosystems, Foster City, CA, USA). Purified cDNA was used as the template for RT-qPCR conducted by using pre-designed primer/probe sets for IFN- $\gamma$, FasL, granzyme B, CXCL9, CXCL10 and $18 \mathrm{~S}$ rRNA (Applied Biosystems) according to the manufacturer's instructions. 18S rRNA was used as an internal control. RT-qPCR was conducted using a LightCycler Rapid Thermal Cycler system (Roche Diagnostic Systems, Inc., Indianapolis, IN, USA).

Cytotoxicity assay. The cytotoxicity assay was performed as previously described (21). For the in vitro cytotoxicity assay, effector cells were obtained from the MLNs of mice with lung metastasis that were stimulated with GalCer and/or LPS. Effector cells were seeded on round-bottom 96-well plates at an effector to target ratio of $20: 1$ with $1.5 \times 10^{5}$ high-labeled target cells. Control cells consisting of $1.5 \times 10^{5}$ low-labeled CT26 tumor cells only were incubated at $37^{\circ} \mathrm{C}$ for $4 \mathrm{~h}$.

$C D 8^{+} T$ cell depletion. Mice were depleted of $\mathrm{CD}^{+} \mathrm{T}$ cells by an intraperitoneally injection of $200 \mu \mathrm{g}$ anti-CD8 mAb (clone 2.43; BioXcell, West Lebanon, NH, USA), respectively, on days 4 and 7 after CT26 injection. Combination therapy was administered on day 5 and 6 following CT26 injection. Nine days after the combination therapy, mice were sacrificed and their lungs were removed to count superficial metastatic nodules.

Statistical analysis. Results are expressed as means \pm SEM. The statistical significance of differences between the experimental groups was analyzed using the Kruskal-Wallis test followed by the Scheffe F-test. Significance was established at $\mathrm{p}<0.05$.

\section{Results}

GalCer and LPS treatment suppresses the growth of tumors in a mouse lung-metastasis model. We examined the antitumor effect of the combination therapy with GalCer and LPS in a mouse lung-metastasis model (Fig. 1A and B). Mice were intravenously injected with $1 \times 10^{5}$ CT26 colon carcinoma cells, and after 5 days, GalCer and LPS were administered to mice presenting with lung metastasis. The mice were then sacrificed to count the tumor metastatic nodules on the lung surface 5 days after treatment. Compared with no treatment or LPS or GalCer monotherapy, the combination therapy with GalCer 
A

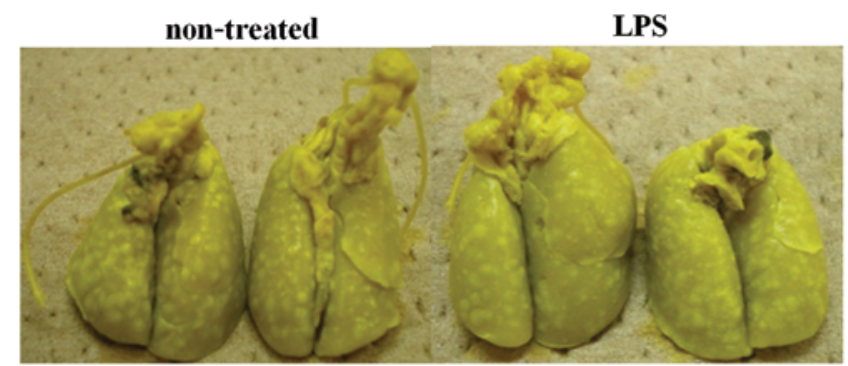

GalCer

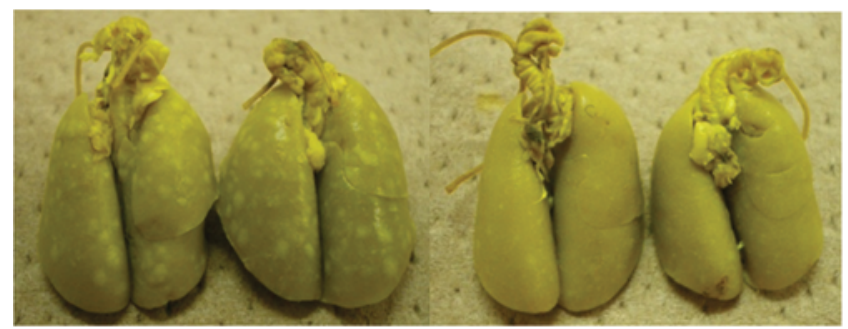

B

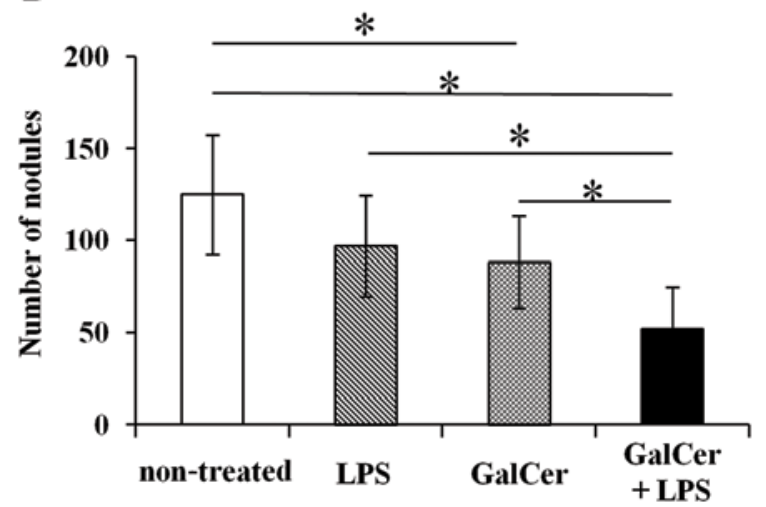

C
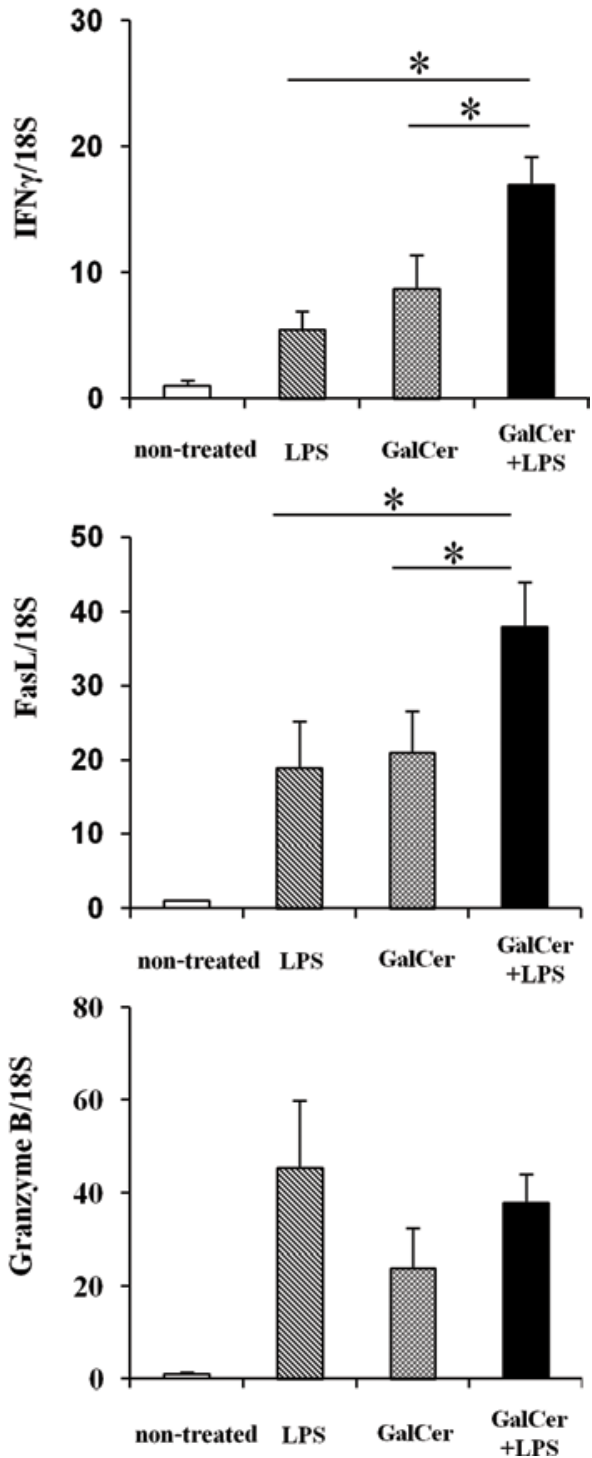

Figure 1. $\alpha$-galactocylceramide (GalCer) and lipopolysaccharide (LPS) combination therapy suppresses tumor growth in a lung metastasis model. (A and B) CT26 colon cancer cells $\left(7.5 \times 10^{4}\right)$ were intravenously injected into BALB/c mice. GalCer ( $\left.1 \mu \mathrm{g} / \mathrm{mouse}\right)$ was intraperitoneally administered 5 days after CT26 cell injection. After $24 \mathrm{~h}$, LPS ( $1 \mu \mathrm{g} / \mathrm{mouse})$ was administered intravenously. Five days following LPS treatment, mice were sacrificed, and their lungs were removed to count tumor metastasis nodules. (C) mRNA expression of interferon- $\gamma$ (IFN- $\gamma$ ), FasL and granzyme B in the lung tissue was analyzed by performing RT-qPCR and was determined on the basis of $18 \mathrm{~S}$ rRNA expression. The data are presented as the means \pm SEM of the results of 5 mice in each group. ${ }^{*} \mathrm{p}<0.05$.

and LPS significantly reduced the number of nodules on the lungs. Next, the mRNA expression levels of IFN- $\gamma$, FasL and granzyme B in the lungs of tumor-bearing mice treated with LPS and/or GalCer were measured by performing real-time RT-qPCR (Fig. 1C). The combination treatment with LPS and GalCer significantly enhanced the mRNA expression of IFN- $\gamma$ and FasL in the lungs of the mice.

Combination therapy with GalCer and LPS enhances tumor antigen-specific cellular immunity. To clarify the mechanism by which GalCer and LPS augment antitumor activity, cells were isolated from the MLNs of CT26 tumor-bearing mice. The cells were cultured with the AH-1 peptide, which is a CD8-restricted epitope expressed by CT26. We observed that the number of IFN- $\gamma$-secreting MLN cells from the mice treated with GalCer and LPS was significantly increased compared to that of mice treated with GalCer or LPS alone (Fig. 2A). In contrast, the cells from the lung tissue of mice exposed to any treatment secreted IFN- $\gamma$ upon stimulation with the AH-1 peptide (Fig. 2B). The MLN cells of mice treated with both GalCer and LPS displayed increased tumor-specific cytotoxicity compared to the MLN cells of the mice treated with GalCer or LPS alone (Fig. 2C). Thereby, the augmentation of the antitumor effect by the combination therapy was induced by a tumor antigen-specific cellular immune response in the MLNs.

Increased $C D 8^{+}$T cell number in the MLNs following GalCer and LPS stimulation. We subsequently examined the phenotype of the lymphocytes in the MLNs of mice treated with GalCer and/or LPS. The $\mathrm{CD}^{+} \mathrm{T}$ cell count in the MLNs from mice co-treated with GalCer and LPS was significantly 
A

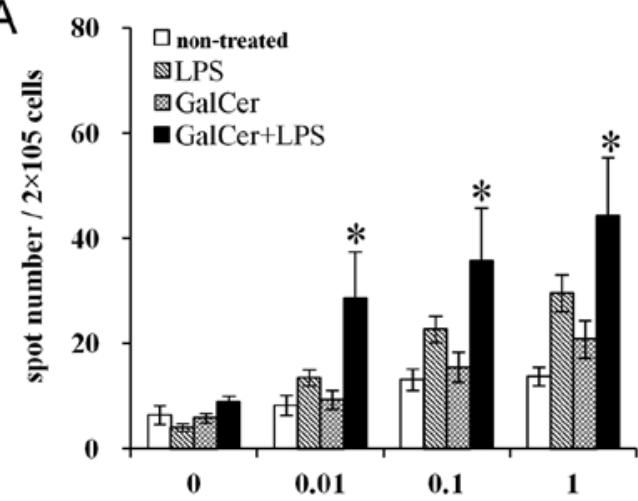

peptide concentration $(\mu \mathrm{g} / \mathrm{ml})$
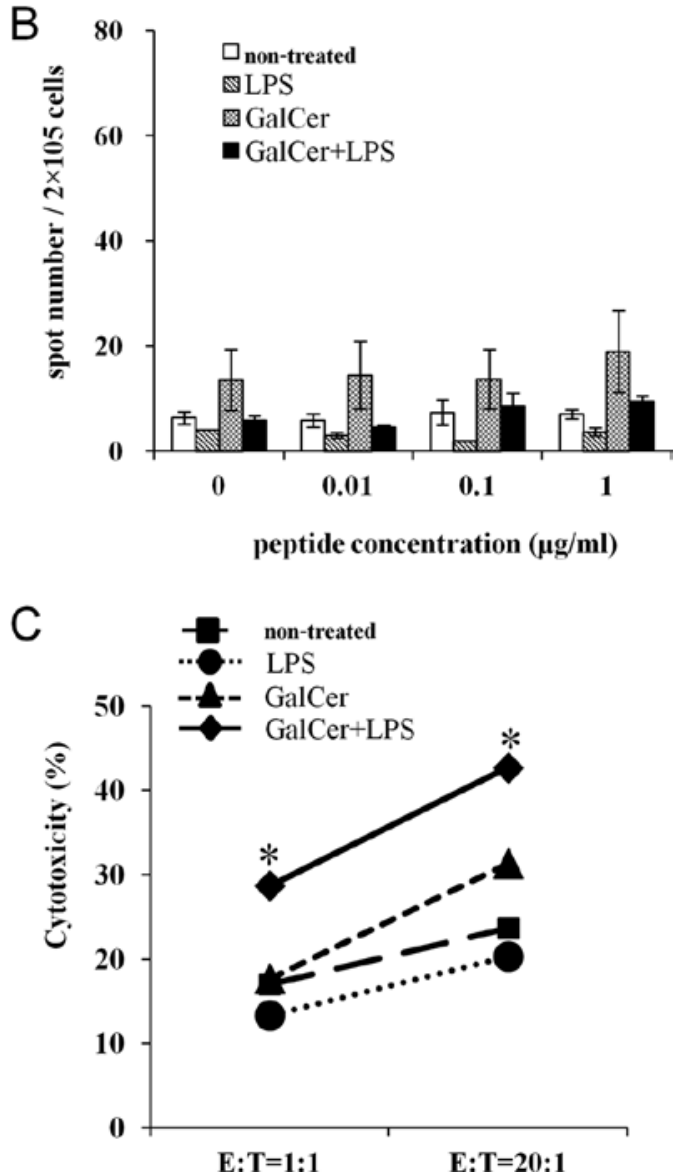

Figure 2. $\alpha$-galactocylceramide (GalCer) and lipopolysaccharide (LPS) combination therapy enhances tumor antigen-specific immune responses. CT26 cells were intravenously injected into BALB/c mice. GalCer $(1 \mu \mathrm{g} /$ mouse $)$ was intraperitoneally administered 7 days after tumor cell injection. After $24 \mathrm{~h}$, LPS ( $1 \mu \mathrm{g} /$ mouse) was administered intravenously. Single-cell suspensions were obtained from (A) mediastinal lymph nodes (MLNs) and (B) lung tissue $24 \mathrm{~h}$ after LPS injection. The cells were stimulated with AH-1 peptide $(0-1 \mu \mathrm{g} / \mathrm{ml})$ in vitro, and interferon- $\gamma(\mathrm{IFN}-\gamma)$ production by the cells was determined using the ELISPOT assay. (C) Mediastinal lymphocytes treated with GalCer and/or LPS were incubated for $4 \mathrm{~h}$ with CFSE-labeled target cells (CT26 cells) at the indicated effector to target ratios (E:T). The mean percentage of specific lysis was calculated as described in Materials and methods. The data are presented as the means \pm SEM of the results of 5 mice in each group. " $\mathrm{p}<0.05$.

increased when compared to the cell count in the non-treated mice or mice treated with GalCer or LPS alone (Fig. 3A). There was no significant difference in $\mathrm{CD}^{+} \mathrm{T}$ cell number
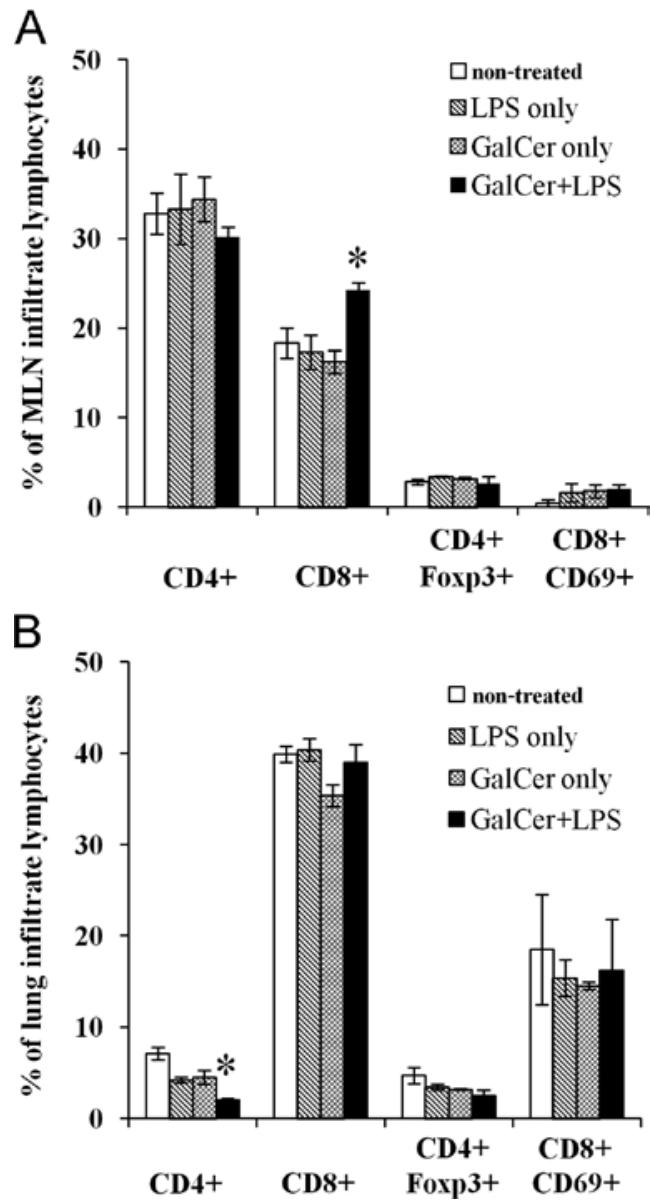

Figure 3. $\alpha$-galactocylceramide (GalCer) and lipopolysaccharide (LPS) combination therapy increases $\mathrm{CD} 8^{+} \mathrm{T}$ cell numbers in mediastinal lymph nodes (MLNs). CT26 cells were intravenously injected into BALB/c mice. GalCer $(1 \mu \mathrm{g} /$ mouse) was intraperitoneally administered 7 days after tumor cell injection. After $24 \mathrm{~h}$, LPS ( $1 \mu \mathrm{g} /$ mouse) was administered intravenously. Single-cell suspensions were obtained from MLNs (A) and lung tissue (B) The cells were stained with anti-CD4, anti-CD8, anti-Foxp3, anti-CD69 antibodies and analyzed using flow cytometry. The data are presented as the means \pm SEM of the results of 5 mice in each group. ${ }^{*} \mathrm{p}<0.05$.

infiltrating the lungs (Fig. 3B). The increase in $\mathrm{CD}^{+} \mathrm{T}$ cell number in the MLNs of the mice co-treated with GalCer and LPS may contribute to the enhancement of tumor antigenspecific immune responses in tumor-bearing hosts.

Expression of chemokine $m R N A$ and chemokine receptors in MLNs stimulated by GalCer and LPS. GalCer and LPS combination therapy or GalCer monotherapy enhanced chemokine mRNA expression in the MLNs (Fig. 4A and B). Although GalCer treatment induced mRNA expression of CXCL9 and CXCL10 in the MLNs, GalCer monotherapy did not markedly enhance the antitumor activity in the mouse lung-metastasis model. We hypothesized that the degree of chemokine receptor expression differed between treatment with GalCer alone and the co-treatment with GalCer and LPS. CXCR3 is the chemokine receptor for CXCL9 and CXCL10, and is mainly expressed on $\mathrm{CD}^{+} \mathrm{T}$ and NK cells. Therefore, we determined the number of CD8 and CXCR3 double-positive T cells in the MLNs by performing flow cytometric analysis. The expression of CXCR3 in the MLNs was increased following GalCer and LPS combination therapy compared to the expression in 
A
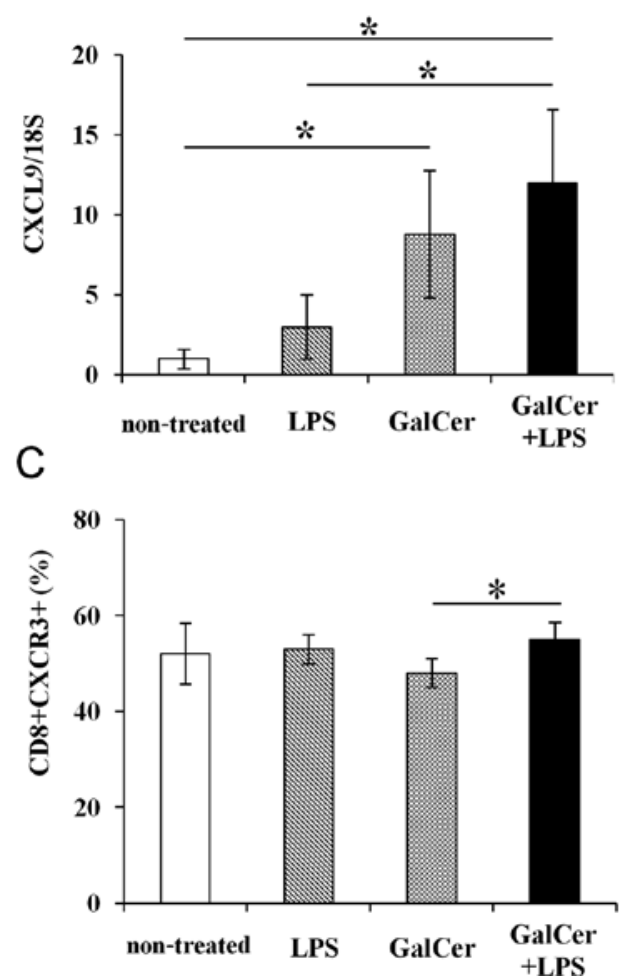

B
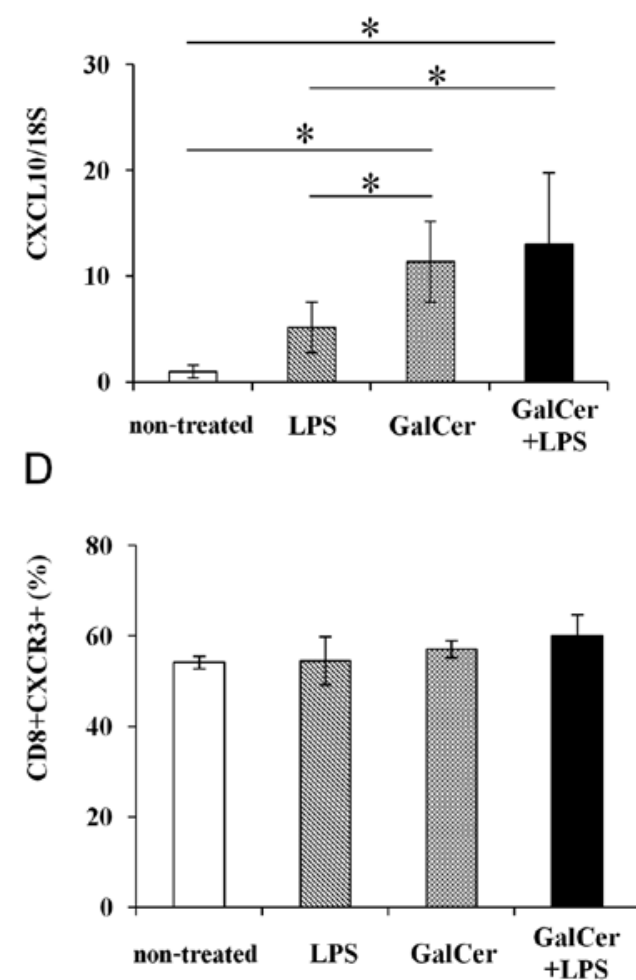

Figure 4. $\alpha$-galactocylceramide (GalCer) and lipopolysaccharide (LPS) combination therapy enhances the expression of chemokine mRNA and chemokine receptors. CT26 cells were intravenously injected into BALB/c mice. GalCer ( $1 \mu \mathrm{g} / \mathrm{mouse})$ was intraperitoneally administered 7 days after tumor cell injection. After $24 \mathrm{~h}$, LPS (1 $\mu \mathrm{g} /$ mouse) was administered intravenously. The mRNA expression of (A) CXCL9 and (B) CXCL10 in the mediastinal lymph nodes (MLNs) from treated mice was analyzed by performing RT-qPCR and was determined on the basis of $18 \mathrm{~S}$ rRNA expression. The data were calculated with reference to the mRNA levels of the respective tissues in control mice. CD8 and CXCR3 double-positive T cells were detected in the MLNs (C) and spleen (D). Data are presented as the means \pm SEM of the results of 5 mice in each group. ${ }^{*} \mathrm{p}<0.05$.

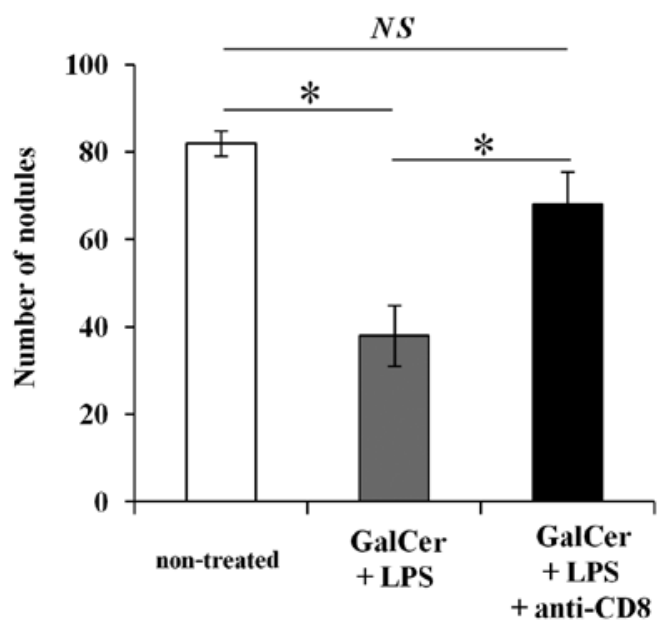

Figure 5. $\mathrm{CD}^{+} \mathrm{T}$ cells are essential for $\alpha$-galactocylceramide (GalCer) and lipopolysaccharide (LPS) combination therapy. CT26 colon cancer cells $\left(7.5 \times 10^{4}\right)$ were intravenously injected into BALB/c mice. Tumor-bearing mice were depleted of $\mathrm{CD}^{+} \mathrm{T}$ cells by administration of anti-CD8 mAb 4 and 7 days following CT26 injection. GalCer ( $1 \mu \mathrm{g} /$ mouse) was intraperitoneally administered 5 days following the CT26 cell injection. After $24 \mathrm{~h}, \mathrm{LPS}(1 \mu \mathrm{g} /$ mouse) was administered intravenously. Five days after LPS treatment, mice were sacrificed and their lungs were removed to count the tumor metastatic nodules. Data are presented as the means \pm SEM of the results of 3 mice in each group. ${ }^{\mathrm{p}}<0.05$. NS, not significant.

the MLNs following treatment with GalCer alone (Fig. 4C). However, there was no difference in the expression of CXCR3 on the spleen between the combination therapy and the treatment of GalCer alone (Fig. 4D). Thus, GalCer treatment induced the production of chemokines, and the combination therapy maintained the CXCR 3 expression in $\mathrm{CD}^{+} \mathrm{T}$ cells. The enhancement of CXCL9 and CXCL10 expression in MLNs and CXCR3 expression on $\mathrm{CD}^{+} \mathrm{T}$ cells resulted in increased $\mathrm{CD}^{+} \mathrm{T}$ cell numbers in the MLNs.

Increased $C D 8^{+} T$ cells are essential for GalCer and LPS combination therapy. To determine whether the increase in $\mathrm{CD}^{+} \mathrm{T}$ cells are important for the observed inhibition of lung metastasis by the combination therapy with GalCer and LPS, we performed anti-CD8 Ab depletion experiment. As shown in Fig. 5, the depletion of $\mathrm{CD}^{+} \mathrm{T}$ cells in the combination therapy restored tumor metastasis nodules. These data indicated that the antitumor effect of the combination therapy with GalCer and LPS was dependent on $\mathrm{CD}^{+} \mathrm{T}$ cells.

\section{Discussion}

In the present study, we demonstrated that co-treatment with GalCer and LPS enhanced tumor antigen-specific immune responses in MLNs and suppressed tumor development in a mouse model of lung metastasis. The increase in $\mathrm{CD}^{+} \mathrm{T}$ cell numbers in MLNs contributed to the enhancement of tumor antigen-specific immune responses. Moreover, our findings illustrated that co-treatment with GalCer and LPS enhanced 
chemokine and chemokine receptor expression in MLNs and stimulated the recruitment of $\mathrm{CD}^{+} \mathrm{T}$ cells to the MLNs of tumor-bearing mice.

Our previous studies revealed that co-treatment with GalCer and a TLR agonist induced strong immune responses in vitro and in vivo. In particular, GalCer and LPS co-treatment strongly enhanced IFN- $\gamma$ production in splenocytes in vitro (18). In the present study, co-treatment with GalCer and LPS enhanced mRNA expression of IFN- $\gamma$ in the lungs of tumor-bearing mice. It is well known that the activation of IFN- $\gamma$ production induces Th1 responses in hosts $(6,19,20)$. Upon CD8 activation, NK and NKT cells can produce IFN- $\gamma$, and these cells exert cytotoxic activity against pathogens, virus-infected and tumor cells (22-24). Many basic and clinical studies have evaluated IFN- $\gamma$ production by host immune cells stimulated with tumor antigens to determine the effects of various therapies in tumorbearing hosts (25-27). Therefore, the enhancement of IFN- $\gamma$ production leads to the induction of antitumor immunity in tumor-bearing hosts. In the present study, GalCer and LPS combination therapy significantly decreased the number of distinct lung metastatic nodules (Fig. 1A and B). Moreover, the results from the ELISPOT assay demonstrated that the combination therapy enhanced tumor antigen-specific Th1 immune responses in tumor draining lymph nodes (Fig. 2A). However, antigen-specific IFN- $\gamma$-producing cells did not increase in the lung after combination therapy (Fig. 2B). In the lung, alveolar macrophages are made up of a majority of pulmonary lymphocytes. Therefore, the ratio of $\mathrm{CD}^{+} \mathrm{T}$ cells in the lung cells using ELISPOT assay was extremely lower than that in the MLNs. As a result, we may not well detect the production of IFN- $\gamma$ in lung single cell suspension. Moreover, tumor-specific cytotoxicity increased in the tumor-bearing mice treated with GalCer and LPS. Thus, the enhancement of tumor antigen-specific immune responses may have decreased lung metastasis in this model. Furthermore, the expression of IFN- $\gamma$ and FasL was enhanced by the combination therapy in the lungs, and the increase in cytotoxic ability in the lungs led to the suppression of tumor growth. In addition, $\mathrm{CD} 8^{+} \mathrm{T}$ cell numbers were significantly elevated in the MLNs of mice treated with both GalCer and LPS (Fig. 3), and this increase may have been involved in the enhancement of antigen-specific immune responses in the MLNs.

In previous studies, various cytokines and chemokines induced by GalCer or LPS treatment contributed to the induction of antitumor immunity $(12,28,29)$. CXCL9 and CXCL10 chemokines are also important for antitumor immune responses (30-32). CXCL9 and CXCL10 mRNA expression was enhanced by GalCer monotherapy or by the combination therapy (Fig. 4A and B). CXCL9 and CXCL10 are IFN- $\gamma$ inducible antiangiogenic chemokines, and they recruit $\mathrm{CXCR}^{+}$ cells (33-35). In our previous report, co-treatment with GalCer and LPS markedly induced IFN- $\gamma$ production in murine splenocytes (18). The enhancement of IFN- $\gamma$ production by GalCer and LPS appears to be involved in the upregulation of CXCL9 and CXCL10 expression. Furthermore, CXCR3 is the receptor for CXCL9 and CXCL10, and it is expressed on T and NK cells. The expression of CXCR3 is induced by GalCer or LPS via IFN- $\gamma$ stimulation (34). The expression of CXCR3 was higher in the $\mathrm{CD} 8^{+} \mathrm{T}$ cells treated with GalCer and LPS than in the $\mathrm{CD}^{+} \mathrm{T}$ cells treated with GalCer alone.
The number of $\mathrm{CXCR}^{+} / \mathrm{CD}^{+}$cells in the mice treated with GalCer alone was lower than that in the mice treated with GalCer and LPS (Fig. 4C). Combination therapy with GalCer and LPS increased chemokine (CXCL9 and CXCL10) expression without affecting the CXCR3 expression. As a result, $\mathrm{CD}^{+} \mathrm{T}$ cells accumulated in the MLNs. The depletion of $\mathrm{CD}^{+} \mathrm{T}$ cells restored the number of lung nodules (Fig. 5). Thus, $\mathrm{CD}^{+} \mathrm{T}$ cells play a critical role in the development of the antitumor effect induced by GalCer and LPS combination therapy. In a previous study, $\mathrm{CD} 8^{+} \mathrm{T}$ cells contributed to optimal tumor antigen-specific immune response. The progression of tumor growth and metastasis was induced by $\mathrm{CD}^{+} \mathrm{T}$ cell depletion (36). The increased $\mathrm{CD}^{+} \mathrm{T}$ cells in MLNs may be essential for GalCer and LPS combination therapy.

In conclusion, the combination therapy with GalCer and LPS induced a tumor antigen-specific immune response and antitumor activity in an established lung metastasis model. The induction of the tumor antigen-specific immune responses was depended on the upregulation of chemokine mRNA expression by GalCer and the maintenance of CXCR3 expression by the combination therapy. Our findings may provide insight for the design of new techniques to prevent lung metastasis.

\section{Acknowledgements}

The authors thank Dr H.S. for kindly providing CT26 tumor cell lines in the present study. This study was supported by a Grant-in-Aid for Scientific Research (C) (24890080) from the Ministry for Education, Culture, Sports, Science and Technology of Japan.

\section{References}

1. Saga K, Tamai K, Yamazaki T and Kaneda Y: Systemic administration of a novel immune-stimulatory pseudovirion suppresses lung metastatic melanoma by regionally enhancing IFN- $\gamma$ production. Clin Cancer Res 19: 668-679, 2013.

2. Purwar R, Schlapbach C, Xiao S, et al: Robust tumor immunity to melanoma mediated by interleukin-9-producing $\mathrm{T}$ cells. Nat Med 18: 1248-1253, 2012.

3. Kronenberg M: Toward an understanding of NKT cell biology: progress and paradoxes. Annu Rev Immunol 23: 877-900, 2005.

4. Kronenberg M and Gapin L: The unconventional lifestyle of NKT cells. Nat Rev Immunol 2: 557-568, 2002.

5. Taniguchi M, Harada M, Kojo S, Nakayama T and Wakao H: The regulatory role of Vo14 NKT cells in innate and acquired immune response. Annu Rev Immunol 21: 483-513, 2003.

6. Paget C, Chow MT, Duret H, Mattarollo SR and Smyth MJ: Role of $\gamma \delta \mathrm{T}$ cells in $\alpha$-galactosylceramide-mediated immunity. J Immunol 188: 3928-3939, 2012.

7. Tessmer MS, Fatima A, Paget C, Trottein F and Brossay L: NKT cell immune responses to viral infection. Expert Opin Ther Targets 13: 153-162, 2009.

8. Tupin E, Kinjo Y and Kronenberg M: The unique role of natural killer $T$ cells in the response to microorganisms. Nat Rev Microbiol 5: 405-417, 2007.

9. Wu L and Van Kaer L: Natural killer T cells and autoimmune disease. Curr Mol Med 9: 4-14, 2009.

10. Perret R, Sierro SR, Botelho NK, Corgnac S, Donda A and Romero P: Adjuvants that improve the ratio of antigen-specific effector to regulatory $\mathrm{T}$ cells enhance tumor immunity. Cancer Res 73: 6597-6608, 2013.

11. Salaun B, Coste I, Rissoan MC, Lebecque SJ and Renno T: TLR3 can directly trigger apoptosis in human cancer cells. J Immunol 176: 4894-4901, 2006.

12. Davis MB, Vasquez-Dunddel D, Fu J, Albesiano E, Pardoll D and Kim YJ: Intratumoral administration of TLR4 agonist absorbed into a cellular vector improves antitumor responses. Clin Cancer Res 17: 3984-3992, 2011. 
13. Spaner DE and Masellis A: Toll-like receptor agonists in the treatment of chronic lymphocytic leukemia. Leukemia 21: 53-60, 2007.

14. Ochi A, Graffeo CS, Zambirinis CP, et al: Toll-like receptor 7 regulates pancreatic carcinogenesis in mice and humans. J Clin Invest 122: 4118-4129, 2012.

15. Ito H, Koide N, Morikawa A, et al: Augmentation of lipopolysaccharide-induced nitric oxide production by $\alpha$-galactosylceramide in mouse peritoneal cells. J Endotoxin Res 11: 213-219, 2005.

16. Ito $\mathrm{H}$, Koide $\mathrm{N}$, Hassan $\mathrm{F}$, et al: Lethal endotoxic shock using $\alpha$-galactosylceramide sensitization as a new experimental model of septic shock. Lab Invest 86: 254-261, 2006.

17. Ohtaki H, Ito H, Ando K, et al: Va14 NKT cells activated by alpha-galactosylceramide augment lipopolysaccharide-induced nitric oxide production in mouse intra-hepatic lymphocytes. Biochem Biophys Res Commun 378: 579-583, 2009.

18. Ando T, Ito H, Ohtaki $\mathrm{H}$ and Seishima M: Toll-like receptor agonists and alpha-galactosylceramide synergistically enhance the production of interferon-gamma in murine splenocytes. Sci Rep 3: 2559, 2013.

19. Hayakawa Y, Takeda K, Yagita H, et al: Critical contribution of IFN- $\gamma$ and NK cells, but not perforin-mediated cytotoxicity, to anti-metastatic effect of $\alpha$-galactosylceramide. Eur J Immunol 31: 1720-1727, 2001.

20. Marth C, Fiegl H, Zeimet AG, et al: Interferon- $\gamma$ expression is an independent prognostic factor in ovarian cancer. Am J Obstet Gynecol 191: 1598-1605, 2004.

21. Nakagawa Y, Watari E, Shimizu M and Takahashi H: One-step simple assay to determine antigen-specific cytotoxic activities by single-color flow cytometry. Biomed Res 32: 159-166, 2011.

22. Seki S, Nakashima H, Nakashima M and Kinoshita M: Antitumor immunity produced by the liver Kupffer cells, NK cells, NKT cells, and CD8 ${ }^{+} \mathrm{CD} 122^{+} \mathrm{T}$ cells. Clin Dev Immunol 2011: 868345, 2011.

23. Smith $\mathrm{SM}$ and Dockrell HM: Role of $\mathrm{CD} 8^{+} \mathrm{T}$ cells in mycobacterial infections. Immunol Cell Biol 78: 325-333, 2000.

24. Exley MA, Lynch L, Varghese B, Nowak M, Alatrakchi N and Balk SP: Developing understanding of the roles of CD1drestricted $\mathrm{T}$ cell subsets in cancer: reversing tumor-induced defects. Clin Immunol 140: 184-195, 2011.

25. Moreno M, Molling JW, von Mensdorff-Pouilly S, et al: IFN- $\gamma-$ producing human invariant NKT cells promote tumor-associated antigen-specific cytotoxic T cell responses. J Immunol 181: 2446-2454, 2008.
26. Wang D, Precopio M, Lan T, et al: Antitumor activity and immune response induction of a dual agonist of Toll-like receptors 7 and 8. Mol Cancer Ther 9: 1788-1797, 2010.

27. Lim JY, Gerber SA, Murphy SP and Lord EM: Type I interferons induced by radiation therapy mediate recruitment and effector function of $\mathrm{CD}^{+} \mathrm{T}$ cells. Cancer Immunol Immunother 63: 259-271, 2014.

28. Choi DH, Kim KS, Yang SH, et al: Dendritic cell internalization of $\alpha$-galactosylceramide from CD8 T cells induces potent antitumor CD8 T-cell responses. Cancer Res 71: 7442-7451, 2011.

29. Chang DH, Osman K, Connolly J, et al: Sustained expansion of NKT cells and antigen-specific T cells after injection of $\alpha$-galactosyl-ceramide loaded mature dendritic cells in cancer patients. J Exp Med 201: 1503-1517, 2005.

30. Andersson A, Yang SC, Huang M, et al: IL-7 promotes CXCR3 ligand-dependent $\mathrm{T}$ cell antitumor reactivity in lung cancer. $\mathrm{J}$ Immunol 182: 6951-6958, 2009.

31. Wong JL, Berk E, Edwards RP and Kalinski P: IL-18-primed helper NK cells collaborate with dendritic cells to promote recruitment of effector $\mathrm{CD}^{+} \mathrm{T}$ cells to the tumor microenvironment. Cancer Res 73: 4653-4662, 2013.

32. Wang P, Yang X, Xu W, Li K, Chu Y and Xiong S: Integrating individual functional moieties of CXCL10 and CXCL11 into a novel chimeric chemokine leads to synergistic antitumor effects: a strategy for chemokine-based multi-target-directed cancer therapy. Cancer Immunol Immunother 59: 1715-1726, 2010.

33. Wallace KL, Marshall MA, Ramos SI, et al: NKT cells mediate pulmonary inflammation and dysfunction in murine sickle cell disease through production of IFN- $\gamma$ and CXCR3 chemokines. Blood 114: 667-676, 2009.

34. Müller M, Carter S, Hofer MJ and Campbell IL: Review: the chemokine receptor CXCR3 and its ligands CXCL9, CXCL10 and CXCL11 in neuroimmunity - a tale of conflict and conundrum. Neuropathol Appl Neurobiol 36: 368-387, 2010.

35. Rosenblum JM, Shimoda N, Schenk AD, et al: CXC chemokine ligand (CXCL) 9 and CXCL10 are antagonistic costimulation molecules during the priming of alloreactive T cell effectors. J Immunol 184: 3450-3460, 2010.

36. Takeshima $\mathrm{T}$, Chamoto $\mathrm{K}$, Wakita $\mathrm{D}$, et al: Local radiation therapy inhibits tumor growth through the generation of tumorspecific CTL: its potentiation by combination with Th1 cell therapy. Cancer Res 70: 2697-2706, 2010. 